\title{
ANÁLISIS DE LA OFERTA Y LA DEMANDA DE LAS CARRERAS UNIVERSITARIAS DE TURISMO Y HOTELERÍA. FORTALEZAS Y DEBILIDADES. CASO DE ESTUDIO ECUADOR
}

\author{
Patricia Carrera Burneo \\ Escuela de Hotelería y Turismo \\ Pontificia Universidad Católica del Ecuador
}

\section{Resumen}

La universidad tiene como misión la educación y formación de talento humano y la generación de nuevos conocimientos, dos requisitos esenciales para el desarrollo económico y social de los pueblos. Se reconoce que el turismo es parte importante de la economía a nivel mundial, y para el Ecuador es primordial para el cambio de su matriz productiva. La mejora permanente de la calidad de la educación superior en turismo, a través de una gestión curricular coherente y pertinente con la realidad del sector, impulsará su desarrollo en el Ecuador.

Este estudio contribuye al análisis de la oferta y la demanda de las carreras de turismo y hotelería por parte de las universidades del Ecuador. Determina la cobertura de éstas y su pertinencia a nivel nacional y, en especial, en la ciudad de Quito. Finalmente, caracteriza la estructura curricular de las carreras y explica sus fortalezas y debilidades, con el objetivo de contribuir con criterios para mejorar su calidad académica.

Palabras clave: Educación, Turismo y Hotelería. 


\title{
ANALYSIS OF OFFER AND DEMAND OF COLLEGE CAREERS ABOUT TOU- RISM AND HOSPITABILITY MANAGEMENT IN ECUADOR
}

\begin{abstract}
Besides generating new knowledge, the mission of every University is educating and shaping human talent. These last aspects are essential requirements for the economic and social development of every society. It has been recognized that tourism is an important component of economy worldwide. Ecuador is not the exception and tourism has become one of the most important components to tackle economy diversification. Therefore, addressing permanent improvements in higher education programs in tourism- based on the needs of the tourism sector- will contribute for the development of the tourism sector in Ecuador. In order to address that, this study examines the existing careers in tourism and hospitability management at universities in Ecuador and analyses the supply and demand for these careers. In doing so, the paper determines the scope of these careers and their national relevance, especially in the city of Quito. The study also characterizes the structure of the curriculum of the examined careers and explains their strengths and weaknesses in order to improve their academic quality.
\end{abstract}

Key words: Education, Tourism and Hospitability. 


\section{INTRODUCCIÓN}

El sector turístico a nivel mundial y en el Ecuador ha experimentado cambios radicales. El turista es consciente de que el descanso, la recreación y el ocio son un derecho, y tiene a disposición medios de comunicación que le permiten estar más informado; por lo tanto exige calidad en el destino, producto y servicio, lo que determina cómo debe funcionar el sistema turístico de cada país.

En el Ecuador durante el 2010 ingresaron un millón de turistas extranjeros, en el 2014 aproximadamente un millón quinientos. Así, el turismo se ubica en el cuarto puesto de las exportaciones no petroleras: contribuyó en el 2013 con mil doscientos millones de dólares (MINTUR, 2015), y en el 2012 aportó con el 4,6\% del total de empleos (WEF, 2013). La ciudad de Quito posee el centro histórico mejor preservado de América Latina. En 1978 la UNESCO lo declaró "Patrimonio Cultural de la Humanidad" por la riqueza histórica y el legado cultural que posee. Por ello y porque ha mejorado en la calidad de sus servicios, es un destino turístico reconocido a nivel mundial que ha experimentado desde el 2008 un incremento sustancial en el número de turistas.

Los datos estadísticos y proyecciones económicas, realizadas por los organismos encargados del turismo en el Ecuador, determinan que este sector crece sostenidamente. Por esta razón, el gobierno ecuatoriano pretende transformar la matriz productiva del país a través de la diversificación de su producción, que se basa en el desarrollo de 14 sectores productivos, entre ellos el turismo (SENPLADES, 2013).

El cambio de una matriz productiva exige la interacción de varios sectores. Y, sin duda, la educación es uno de los pilares fundamentales para que un pueblo se desarrolle. Estudios económicos y sociales han comprobado que existe una relación directa entre el mejoramiento de la calidad de la educación y la reducción de los niveles de pobreza y el crecimiento económico de una sociedad. Una buena educación se traduce en el incremento de las tasas de innovación, propicia una participación activa en la sociedad, entre otros indicadores sociales (Osorio et al., 2012). Cañibano Sánchez (2005, p. 257) expresa que "cualquier economía, desde la más tradicional o rudimentaria hasta la más interconectada y tecnológica, es necesariamente una economía basada en el conocimiento de los individuos y agrupaciones que la componen."

Si bien es cierto que la calidad de la educación es muy importante, también lo son la cobertura, pertinencia y los niveles de educación y formación, condiciones sine qua non para el desarrollo social y económico de un pueblo. 
El reto está planteado para el Ecuador, el gobierno actual requiere que el sector turístico se desarrolle de manera sustentable, y la Universidad debe ser parte de este cambio. Por lo tanto, es imprescindible la mejora de la calidad de la educación superior, proceso que se encuentra en marcha, y para lo cual se requiere de una mayor participación y cohesión entre el gobierno, la academia y la empresa privada.

Este estudio tiene como objetivo analizar la oferta y la demanda de las carreras de turismo y de hotelería a nivel nacional, con énfasis en Quito, para determinar las fortalezas y debilidades que las caracterizan y contribuir con criterios para mejorar la estructura curricular de las carreras.

\section{2. ¿A QUÉ OBEDECE LA HETEROGENEIDAD DE LAS CARRERAS DE TURISMO EN LATINOAMÉRICA Y EN EL ECUADOR? TENDENCIAS INTER- NACIONALES SOBRE LA GESTIÓN CURRICULAR EN TURISMO}

En el Ecuador como en otros países latinoamericanos, la educación superior ha transitado por las tres reformas citadas por Rama (2006). La primera fue marcada por la reforma de Córdoba, en 1818, que promovió la expansión de universidades públicas, se instauró un modelo público, laico, gratuito, cogobernado y autonomista y que se prolongó hasta inicios de los setenta. La segunda reforma (Rama, 2006, p. 12) se caracterizó por la presencia de un modelo universitario binario, público-privado, y por la mercantilización de la educación superior. Y la tercera reforma (Rama, 2006, p. 13) definida por un modelo trinario: público-privado-internacional, en la que se observan procesos de masificación e internacionalización.

La segunda reforma, que en algunos países latinoamericanos se extendió hasta mediados de los 90 (Rama, 2006, p. 12) y en Ecuador hasta la década del 2000, tuvo una marcada influencia en la educación superior, no solo en el ámbito del turismo sino en otros campos profesionales. Predominó una lógica de mercado que se articuló a los intereses privados y mercantiles, convirtiendo a la Universidad en un negocio rentable, por lo que se crearon indiscriminadamente universidades en todo el país (Ramírez, 2013, p. 10-11). Hasta el 12 de abril del 2012, el Ecuador tuvo 71 universidades, 45 de ellas creadas entre 1992 y 2006, lo que evidencia un crecimiento del $273 \%$ en solo 14 años (CEAACES, 2013, p. 11). En lo que se refiere a la educación superior en turismo y hotelería, se encuentran vigentes en el país 117 carreras.

En el estudio de Vandam (2013), luego de analizar ciento tres (103) programas curriculares en turismo y hotelería de diferentes universidades de distintos países de América Latina y Norteamérica, se explica la heterogeneidad de carreras y por ende la disimilitud de la oferta curricular y de los títulos otorgados. Esto obedece, sin duda, a la falta de es- 
se extendió hasta la década de los noventa. Otro suceso que marca sobre todo la disimilitud de las estructuras curriculares de las carreras de turismo, como lo reconoce Vandam (2013), es la unidad académica que les dio origen: se encuentran carreras de turismo y hotelería en facultades como las de Economía, Ciencias Humanas o Lingüística, entre otras.

Las particularidades citadas, la juventud del turismo como disciplina y la investigación multidisciplinaria acrítica (Niding et al., 2011), han perjudicado la construcción epistemológica del turismo (Tribe, 2005; Muñoz de Escalona, 2011) y por ende la consolidación de éste en el ámbito científico. Este impase tiene notable incidencia en el reconocimiento académico de las carreras de turismo y hotelería. En la actualidad se ofertan carreras con un fuerte componente administrativo y otro débil en turismo, lo que no permite entender el turismo ni cómo manejarlo.

Por otro lado, un estudio de la oferta de las carreras en cualquier disciplina del conocimiento exige un análisis, aunque general, de la estructura curricular de éstas. La identificación de los núcleos disciplinares que conforman la estructura curricular determina la orientación profesional de la carrera. Para el caso del turismo y los servicios conexos se identifican tres tendencias a nivel mundial. Autores como Tribe (2005) reconocen al sector como un pilar importante de la economía; por tanto, la reflexión sobre la educación superior en turismo es relevante y hace énfasis en la planificación y gestión curricular. El autor analiza el currículo de algunas carreras y determina que la mayoría son técnicas; propone entonces la creación de "carreras liberales", las que se caracterizan por apartarse del mundo de los negocios, abordando al turismo como un fenómeno socioeconómico.

En estudios más recientes, como el de Ring, Dickinger y Wöber (2009, p. 107-109), se destaca la importancia de desarrollar en los educandos competencias genéricas o transferibles como el pensamiento crítico, la creatividad y la resolución de problemas, manejo de nuevas tecnologías, entre otras, lo que permitirá a los futuros profesionales adaptarse a los constantes cambios del entorno. Para Ring, Dickinger y Wöber (2009), la dicotomía expuesta por Tribe (2005) es relevante y proponen que exista un equilibrio en los currículos de turismo. Es decir, la estructura curricular de las carreras de turismo y hotelería deberá satisfacer las demandas del sector turístico y, al mismo tiempo, estimular la reflexión de los estudiantes para que puedan intervenir en la realidad de este sector proactivamente y no sólo tengan como referente las tendencias del mercado. Por tanto, se enfatiza en un enfoque multidisciplinario que incluya a las ciencias sociales y ambientales, con la finalidad de entender el turismo como un fenómeno global y prever su impacto en la sociedad y el entorno.

El estudio realizado por Wang, Ayres y Huyton (2009) en el entorno australiano, enfatiza en que el currículo debe ser diseñado por los representantes de la industria turística y 
los educadores. Los autores se inclinan por un currículo que desarrolle las competencias técnicas, condición que favorece la inserción laboral de los graduados.

Por lo expuesto, la múltiple oferta de carreras de turismo y hotelería no sólo obedece al mercantilismo impulsado por políticas neoliberales (Ramírez, 2013), sino al desconocimiento de la disciplina como tal. Como sucedió en otros países latinoamericanos, en el Ecuador el turismo y la hospitalidad han sido desarrollados en los últimos cuarenta años por académicos de diferentes áreas disciplinares, lo que originó enfoques curriculares diversos y como consecuencia una oferta muy heterogénea de carreras y titulaciones. Este hecho explica el diagnóstico que realiza Larrea de Granados $(2014$, p. 6) sobre la gestión curricular de las universidades, en el que se identifica como problema para la calidad de la educación superior la diversificación de carreras y la desactualización de los modelos curriculares.

Por estos antecedentes, el Consejo de Educación Superior (CES) emitió en el 2013 el Reglamento de Régimen Académico (RRA) para normalizar la estructura curricular básica que deben observar las carreras que se imparten en las universidades ecuatorianas. En julio del 2014, el CES expidió el Reglamento de armonización de la nomenclatura de títulos con el objeto de establecer normas para armonizar la nomenclatura de títulos profesionales y grados académicos, otorgados por las universidades que forman parte del Sistema de Educación Superior del Ecuador; la nomenclatura se basa en los campos del conocimiento establecidos por la UNESCO (CES, 2014, p. 3).

La meta del presente estudio es determinar cómo está constituida la oferta y la demanda de las carreras de turismo y hotelería en el Ecuador, su cobertura, las tendencias profesionales, su estructura curricular básica, el número de estudiantes a nivel nacional y en especial en Quito, así como el potencial académico que ofrecen para diferenciarse. Además pretende aportar con criterios para la reestructuración del currículo de las carreras de turismo y hotelería de acuerdo con lo dispuesto en los reglamentos ya mencionados.

\section{OBJETIVOS}

- Analizar la oferta y la demanda de las carreras de turismo y hotelería, a nivel nacional e internacional, para identificar las fortalezas y debilidades y aportar con criterios para el rediseño curricular de las carreras.

\section{METODOLOGÍA}

El presente estudio se apoya en la investigación documental. La información sobre las universidades y las carreras que ofertan en los ámbitos del turismo y la hotelería fueron obtenidas de las páginas web de cada institución. 
Se recurrió también a fuentes institucionales rectoras de la educación superior en el Ecuador como el CES y la SENESCYT. De esta última se obtuvo registros del número de estudiantes por universidad a nivel nacional durante el período 2010-2013. En la página web del CEAACES se encuentra información sobre la categorización de las universidades. El Ranking mundial de universidades, elaborado por el Instituto Superior de Shanghái Jiao Tong de la Universidad de China (2015), se utilizó para conocer cuáles son las mejores universidades a nivel latinoamericano, principalmente, y dentro de éstas escoger las que ofertan carreras tanto en turismo como en hotelería.

Con el objetivo de facilitar el análisis de las carreras a nivel nacional, ya que su oferta es en extremo heterogénea, se procedió a sistematizar las titulaciones, agrupándolos por títulos profesionales (licenciatura/ ingeniería); luego se las reunió por denominaciones generales, que abarcan títulos específicos en las ramas que reconoce la SENESCYT: turismo, turismo y hotelería y hotelería; finalmente se identificaron 19 denominaciones generales, en las que se agruparon los títulos otorgados por las 42 universidades a nivel nacional. A continuación se presenta un esquema que describe el proceso para la determinación de las denominaciones generales:

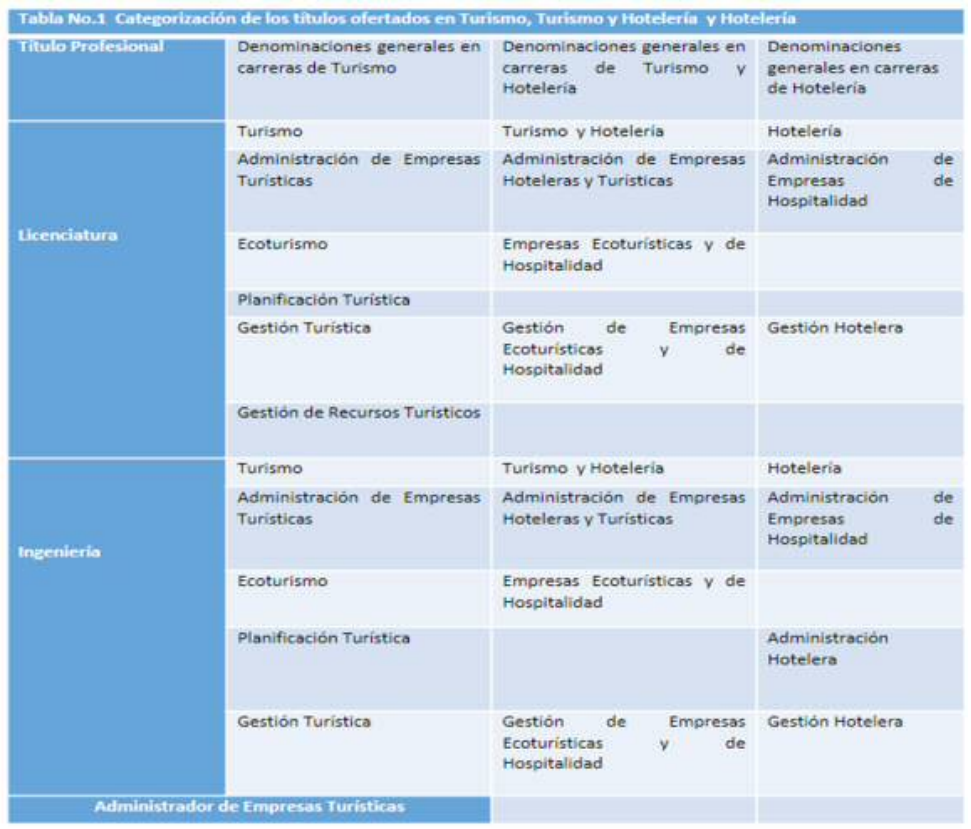

Elaboración propia 
Para conocer los precios aproximados de las carreras en universidades privadas, se priorizó aquellas categorizadas con calificación $\mathrm{B}$, ya que cumplen con estándares de calidad homogéneos y que están localizadas en Quito, ciudad representativa a nivel nacional de la oferta de carreras de turismo y hotelería. Luego se estableció los rangos de precios por universidad, ya que estos varían de acuerdo al número de créditos que toma el estudiante por semestre y a otros aranceles propios de cada universidad.

Para determinar si cada carrera, sus perfiles profesionales, se ajustan con las competencias básicas del profesional en turismo, se desarrolló la siguiente metodología: se resumió los conocimientos, habilidades y actitudes básicas que requiere un profesional en turismo, a partir del análisis realizado por autores como: García Manjón y Pérez López (2008), Wang, Ayres y Huyton (2009) y principalmente de los "Lineamientos de mejora para la formación de recursos humanos en turismo" publicado por el Ministerio de Educación y de Turismo de Argentina (2014). Así se obtuvo 7 descriptores que se compararon con los declarados en el Perfil del egreso o profesional de las carreras de turismo y hotelería de las universidades de categorías A y B de la ciudad de Quito (6); por liderar la educación superior en turismo en la provincia del Guayas, se analizó a la Escuela Politécnica del Litoral; en Chimborazo a la Escuela Politécnica del Chimborazo, y en Azuay a la Universidad de Cuenca. La Universidad de Especialidades Turísticas (Quito, categoría C) fue tomada en cuenta porque se especializa en turismo y afines y observó una demanda considerable. Finalmente, se identificó si cada competencia básica estaba declarada o no en el perfil de egreso o profesional. También se analizaron las mallas curriculares (mesocurrículo) de las universidades mencionadas, y como indicador se obtuvo el porcentaje de materias básicas y de los núcleos disciplinares, tanto en turismo como del profesional/técnico en relación al total de materias de cada carrera.

Los datos descriptivos y cuantitativos extraídos de fuentes secundarias fueron procesados en el programa estadísticos SPSS versión 18.

\section{RESULTADOS}

\subsection{Universidades a nivel nacional que ofertan carreras de turismo y hotelería. Cobertura. Títulos otorgados}

\section{Datos de contexto}

El censo realizado por el Instituto Nacional de Estadísticas y Censos (INEC) en el 2010 reporta, para ese año, 15'012.228 de habitantes. El número de universidades hasta el 2012 fue de 71, la población que estudiaba en éstas era de 719.553 estudiantes $(0,63 \%$ con respecto al total de la población); el 59,43\% estudiaron en IES públicas, el 40,58\% en privadas. 
Conforme a las proyecciones poblacionales del INEC 2015, el Ecuador tiene una población de 16'278.844 habitantes. El número de universidades disminuyó a nivel nacional, después de que el CEAACES (2012) determinó el cierre de 14 universidades de categoría E. Actualmente existen un total de 52 entre públicas, cofinanciadas y privadas (no se tomaron en cuenta para este estudio dos universidades públicas que están intervenidas por el CES: la Universidad Estatal de Guayaquil y la Luis Vargas Torres de Esmeraldas, ni tres más que se han cerrado por causas que no explican sus organismos responsables). De acuerdo con el Informe General sobre la Evaluación, Acreditación y Categorización de las Universidades y Escuelas Politécnicas, aprobado el 26 de noviembre de 2013, en la categoría A se encuentran 4 universidades, en la B: 22 , en la C: 18 y en la D: 8 (CES, 2013).

De las 52 universidades ecuatorianas, 42 ofertan 117 carreras de turismo, hotelería y turismo y hotelería a nivel nacional; el $85 \%$ de las carreras son ofertadas, en su mayoría, por universidades de categoría B (59\%) y C (26\%). Las instituciones de educación superior (IES) públicas representan el 52\%, las privadas el 36\% y cofinanciadas el 12\%.

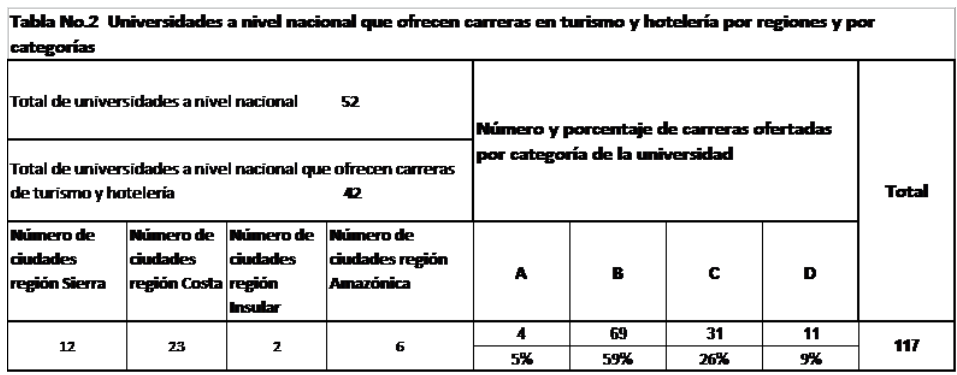

Fuente: CES, 2013.

Elaboración propia

Cobertura de universidades a nivel nacional: De las 42 universidades que ofrecen carreras de turismo y hotelería, 19 tienen extensiones en todo el país. La Universidad Particular de Loja posee 24, ya que tiene modalidad a distancia; continúa la Universidad Católica Santiago de Guayaquil, con 17 extensiones; en tercer lugar se encuentra la PUCE con 7; la Universidad Laica Eloy Alfaro de Manabí con 5 y la Universidad Metropolitana con 4, entre las más representativas. Las universidades y sus respectivas extensiones generan 117 carreras de turismo y hotelería a nivel provincial.

Las universidades se encuentran distribuidas en las 24 provincias que tiene el país. En la región Sierra 12 ciudades cuentan con universidades que ofrecen carreras de turismo y hotelería, en la Costa 23 y en la región Amazónica 6 (ver Tabla No.2). 
En la provincia de Pichincha se ofertan 20 carreras (17,09\%), en Manabí $16(13,68 \%)$ y en Guayas $14(11,97 \%)$. Las tres provincias concentran el 43\% de las 117 carreras a nivel nacional (ver Fig. No.1).

Fig. No.1 Número de carreras de Turismo y Hotelería por provincia (2010-2013).

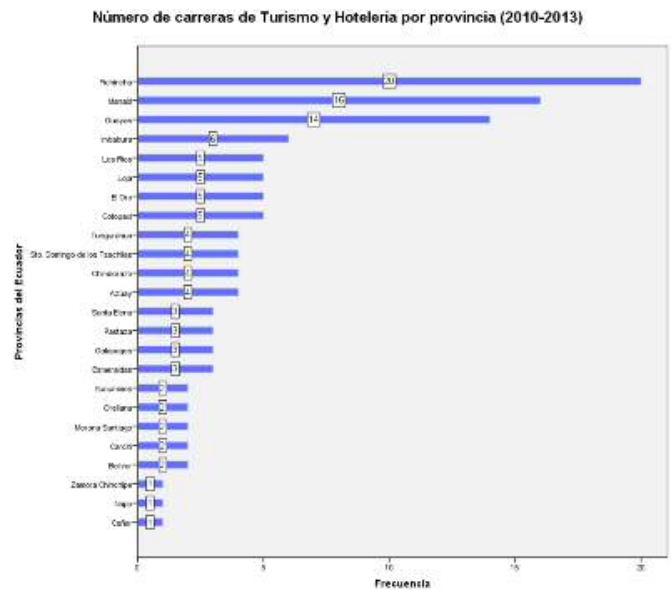

Fuente: SENESCYT (2015).

Elaboración propia.

Títulos de grado: Los títulos están relacionados con las carreras que se ofertan en el Ecuador, cada carrera otorga un título diferente. Para facilitar su análisis, se agruparon en un número de 19 (100\%). El título que se otorga con más frecuencia es la Licenciatura en Turismo (17,65\%), seguido por el de Ingeniería en Ecoturismo (10,29\%); el tercer lugar es compartido por la Ingeniería en Administración de Empresas Turísticas, Ingeniería en Empresas Hoteleras y Turísticas, Ingeniería en Administración Hotelera, cada uno con un $8,82 \%$.

Si bien es cierto que existen títulos como la Licenciatura en Turismo, que son otorgados con una frecuencia considerable; sin embargo, como se observa en la Fig. No.2, sumando todas las variantes del título relacionado con el de Ingeniero(a) o Licenciado(a) en Administración y Gestión de Empresas turísticas y hoteleras, el título que se oferta a nivel nacional con mayor frecuencia tiene relación con la Administración/Gestión de Empresas turísticas y/o hoteleras. 
Fig. No.2 Títulos ofertados (\%) por carrera a nivel nacional

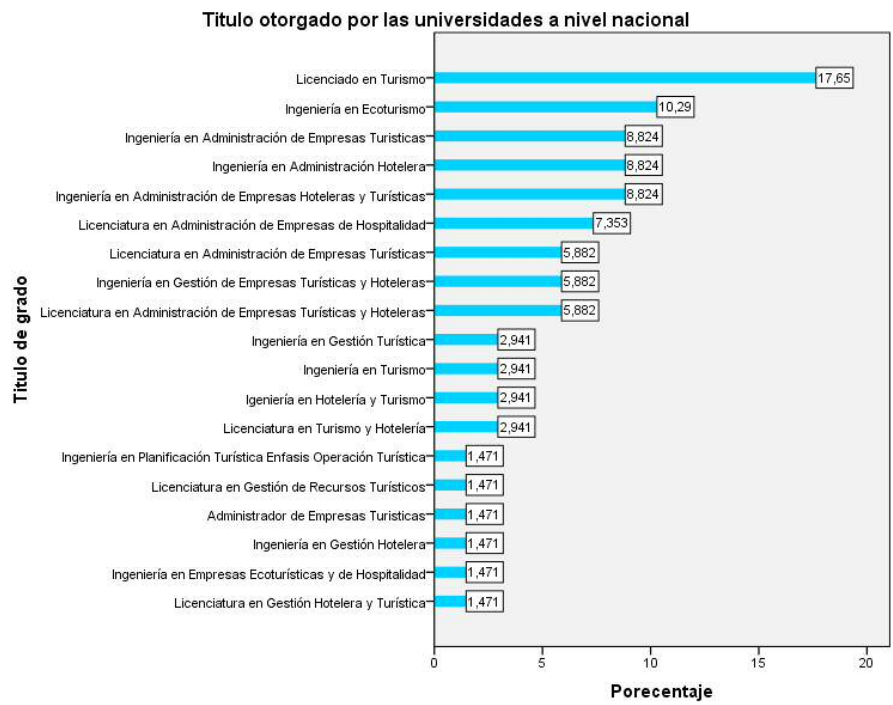

Fuente: SENESCYT (2015).

Elaboración propia.

\subsection{Demanda de estudiantes}

Como se observa en la Tabla No.2, las carreras relacionadas con el turismo, turismo y hotelería y hotelería tienen una demanda nacional que oscila entre 12.500 y 13.600 estudiantes por año, en el período 2010-2013.

De manera más específica, las carreras que corresponden al ámbito turístico son las que más demanda han tenido en el período señalado: cada año se matricularon aproximadamente entre 6.000-8.000 estudiantes. En segundo lugar se encuentran las carreras que ofertan en conjunto turismo y hotelería, que en el mismo período tuvieron entre 3.500-5.400 estudiantes; y en tercer lugar se ubican las carreras de hotelería, con una demanda que fluctuó entre 1.300 y 1.600 estudiantes. 
Tabla No.3 Demanda de estudiantes por tipo de carrera a nivel nacional (2010-2013)

\begin{tabular}{|c|c|c|c|c|}
\hline \multirow{2}{*}{ TIPO DE CARRERA } & \multicolumn{4}{|c|}{ NÚMERO DE ESTUDIANTES /AÑO } \\
\cline { 2 - 5 } & 2010 & 2011 & 2012 & 2013 \\
\hline Turismo & 6.890 & 8.448 & 5.981 & 6.285 \\
\hline $\begin{array}{l}\text { Turismo y } \\
\text { Hotelería }\end{array}$ & 4.493 & 3.520 & 5.059 & 5.459 \\
\hline Hoteleria & 1.681 & 1.645 & 1.514 & 1.366 \\
\hline TOTAL & 13.362 & 13.613 & 12.554 & 13.110 \\
\hline
\end{tabular}

Fuente: SENESCYT (2015).

Elaboración propia

Tabla No.4 Universidades y demanda de estudiantes (\%)

\begin{tabular}{|c|c|c|}
\hline $\begin{array}{l}\text { Nombre de la universidad } \\
\text { (incluye extensiones) }\end{array}$ & $\begin{array}{l}\text { Rangos número } \\
\text { de estudiantes }\end{array}$ & $\begin{array}{c}\text { Demanda } \\
\text { promedio de } \\
\text { estudiantes } \\
\text { porcentaje }(\%)\end{array}$ \\
\hline Univ ersidad Tecnológica Equinocoial (UTE) & \multirow{3}{*}{$600-400$} & \multirow[t]{3}{*}{ ? } \\
\hline Escuela Superior Pilitécnioa del Lit oral (ESPOL) & & \\
\hline Unin ersidad Nacional de Loja (UNL) & & \\
\hline Unin ersidad Técnica de A mbato (UTA) & \multirow{4}{*}{$400-300$} & \multirow{4}{*}{20,2} \\
\hline Univ ersidad de Cuenoa (UCuenoa) & & \\
\hline Univ ersidad Central del Eouador (UCE) & & \\
\hline Unin ersidad de las A méricas (UDA.) & & \\
\hline Univ ersidad de Especialidades Turisticas (UCT) & \multirow{5}{*}{$300-200$} & \multirow{5}{*}{17.5} \\
\hline Univ ersidad Estatal de Quev edo (UEQ) & & \\
\hline Unin ersidad Técnica del Norte (UTGN) & & \\
\hline Escuela Superior Polit écnica A gropecuaria de Manabí & & \\
\hline Escuela Superior Polit écnica de Chimbor az o (ESPOCH) & & \\
\hline Universidad Nacional del Chimborazo (UNCH) & \multirow{13}{*}{$200-100$} & \multirow{13}{*}{26,9} \\
\hline Unin ersidad de las Fuerzas Armadas (UFA) & & \\
\hline Univ ersidad Tecnológica ECOTEC (ECOTEC) & & \\
\hline Unin ersidad Estatal de Milagro (UEM) & & \\
\hline Univ ersidad del Az uay (UDA) & & \\
\hline $\begin{array}{l}\text { Univ ersidad Particular de Es pecialidades Espíritu Santo } \\
\text { (UPEES) }\end{array}$ & & \\
\hline Unin ersidad Laic a Eloy A If aro de Manabí (ULEAM) & & \\
\hline Unin ersidad Tecnológica Israel (UT) & & \\
\hline Univ ersidad Técnioa de Machala (UTM) & & \\
\hline Univ ersidad Estatal Amaz ónioa (UEA) & & \\
\hline Univ ersidad Estatal Península de Santa Eena (UESE) & & \\
\hline Pont ificia Unin er sidad Católica del Eouador (PUCE) & & \\
\hline Univ ersidad de Otavalo (UOT) & & \\
\hline Univ ersidad Técnica de Cotopaxi (UTC) & \multirow{17}{*}{1000} & \multirow{17}{*}{13.9} \\
\hline Univ ersidad Estatal de Bolin ar (UEB) & & \\
\hline Unin ersidad Politécnica Estatal del Carchi (LFEC) & & \\
\hline Unin ersidad Regional Autónoma de los A ndes (URAA) & & \\
\hline Univ ersidad Particular Int ernacional SE $<$ (SE $K$ ) & & \\
\hline Unin ersidad Particular San Gregor io de Portov iejo & & \\
\hline Unin ersidad Téonica de Babahoyo (UTB) & & \\
\hline Univ ersidad Iber oamericana del Ecuador (UIE) & & \\
\hline Univ ersidad Técnic a Particular de Loja (UTPL) & & \\
\hline Unin ers idad Internacional del Ecuador (UIE) & & \\
\hline Univ ersidad Estatal del Sur de Manabí (UESM) & & \\
\hline Unin ersidad Cat ólica de Santiago de Guay aquil (UCSG) & & \\
\hline Unin ersidad Metropolit ana (UM) & & \\
\hline Univ ersidad Teonológica Empresarial de Guay aquil & & \\
\hline Univ ersidad San Francisco de Quito (USFQ) & & \\
\hline Unin ersidad de los Hemisferios (UDH-) & & \\
\hline Universidad del Pacif ico Escuela de Negocios (UP) & & \\
\hline Promedio de estudiantes (2010-2013) & 13159,75 & 1009 \\
\hline
\end{tabular}

Fuente: SENESCYT (2015).

Elaboración propia 
Del análisis de la Tabla No.4, se puede señalar que tres universidades (UTE-ESPOLUNL) concentraron en promedio el 22\% de estudiantes durante el período 2010-2013; la SENESCYT (2015) reportó una fluctuación entre 600-400 estudiantes. Un segundo grupo de universidades (UTA, U. Cuenca, UCE, UDLA) tuvieron una demanda que osciló entre 400-300 estudiantes por año (período 2010-2013), y absorbieron en promedio el $20 \%$ de la demanda. Un tercer grupo (13 universidades), cuya demanda varió entre 200-100 estudiantes al año (período 2010-2013), aglutinaron en promedio el $27 \%$ de estudiantes. Es decir que el $48 \%$ del total de universidades que ofrecen carreras de turismo y hotelería concentran en promedio el $70 \%$ de la demanda a nivel nacional; 12 son públicas, 5 privadas y 3 cofinanciadas.

\subsection{Oferta y demanda de carreras en la ciudad de Quito}

En Quito se encuentran 15 universidades (36\%) que ofertan carreras de turismo y hotelería; 7 de éstas se encuentran en la categoría B, 6 en la C y 2 en la A. La mayoría de universidades (11) son privadas, 2 públicas y 2 cofinanciadas.

En relación a la concentración de la demanda en Quito, es necesario aclarar que durante el 2010 la UTPL registró 352 estudiantes, dato que eleva el porcentaje de la demanda al 31\%; en los años subsiguientes la universidad en mención no registra estudiantes. De acuerdo con los datos del período 2011-2013, las universidades de Quito cubrieron entre el $25-27 \%$ de la demanda a nivel nacional. Tanto la UTE (cofinanciada) como la UCE (pública) atendieron el 49\% de la demanda de estudiantes de Quito. El conjunto de universidades (6): UDLA, UFA, UCT, PUCE, UTI, y UM, observaron una demanda promedio del $41 \%$, y las últimas 7 el 10\% restante. La demanda de la PUCE se encuentra entre el 8 y $11 \%$ (ver Tabla No.5).

\subsubsection{Precios de las universidades de la ciudad de Quito}

En la Tabla No.6 se describen los precios aproximados de las distintas universidades privadas en Quito, los que oscilan entre 1.500 y 5.000 USD. Cada universidad, independientemente de su categoría, fija el costo de la matrícula y de otros aranceles que tienen relación con el uso de servicios como biblioteca, laboratorios, entre otros

\subsection{Perfiles de egreso, núcleos disciplinares, prácticas pre-profesionales, vinculación con la sociedad, investigación y publicaciones}

La mayoría de los perfiles de egreso, tanto de las carreras de turismo como de hotelería de las distintas universidades de Quito y otras analizadas, describen 6 de las 7 competencias básicas acerca de los conocimientos, habilidades y actitudes requeridas por el profesional para desempeñarse en el sector turístico y hotelero. La habilidad de desempeñarse con 
efectividad en grupos multidisciplinarios es citada sólo en 2 de las 10 universidades; actuar con espíritu emprendedor y aprender en forma autónoma y continúa, se menciona en los perfiles de 5 universidades (ver Tabla No.7).

\section{Tabla No.5 Número de estudiantes en las diferentes universidades de la ciudad de Quito (2010-2013)}

\begin{tabular}{|c|c|c|c|c|c|c|}
\hline \multirow{2}{*}{ Nombre de la universidad } & \multirow{2}{*}{ Ciudad } & \multirow[b]{2}{*}{ Categoría } & \multicolumn{4}{|c|}{ Año } \\
\hline & & & 2010 & 2011 & 2012 & 2013 \\
\hline Universidad Tecnológica Equinoccial (UTE) & Quito & B & 1.293 & 1.192 & 1.140 & 1.039 \\
\hline Universidad Central del Ecuador (UCE) & Quito & B & 719 & 459 & 496 & 682 \\
\hline Universidad de las Américas (UDLA) & Quito & c & 268 & 329 & 343 & 338 \\
\hline Universidad de las Fuerzas Armadas (UFA) & Quito & A & 298 & 309 & 257 & 373 \\
\hline Universidad de Especialidades Turisticas (UCT) & Quito & c & 417 & 218 & 291 & 262 \\
\hline Pontificia Universidad Católica del Ecuador (PUCE) & Quito & B & 160 & 286 & 340 & 373 \\
\hline Universidad Tecnológica Israel (UTI) & Quito & c & 153 & 163 & 111 & 86 \\
\hline Universidad Metropolitana (UM) & Quito & c & 148 & 96 & 96 & 99 \\
\hline Universidad Técnica Particular de Loja (UTPL) & Quito & B & 352 & 0 & 0 & 0 \\
\hline Universidad Internacional del Ecuador (UIE) & Quito & c & 95 & 90 & 58 & 90 \\
\hline Universidad Particular Internacional SEK (SEK) & Quito & B & 85 & 95 & 70 & 47 \\
\hline Universidad Iberoamericana del Ecuador (UIE) & Quito & B & 73 & 72 & 48 & 62 \\
\hline Universidad San Francisco de Quito (USFQ) & Quito & A & 57 & 47 & 38 & 22 \\
\hline Universidad de los Hemisferios (UDH) & Quito & B & 12 & 8 & 10 & 9 \\
\hline Universidad del Pacifico Escuela de Negocios (UPN) & Quito & c & 7 & 8 & 3 & 0 \\
\hline Total de estudiantes & & & 4.137 & 3.372 & 3.301 & 3.482 \\
\hline $\begin{array}{r}\text { Porcentaje de estudiantes en relación al total } \\
\text { nacional de cada año }\end{array}$ & & & $31 \%$ & $25 \%$ & $26 \%$ & $27 \%$ \\
\hline
\end{tabular}

Fuente: SENESCYT (2015).

Elaboración propia

Tabla No.6 Precios en las universidades privadas de Quito

\begin{tabular}{|c|c|c|}
\hline Categoría & Universidad & $\begin{array}{l}\text { Precio aproximado USD por } \\
\text { semestre }\end{array}$ \\
\hline A & $\begin{array}{l}\text { Universidad San Francisco de } \\
\text { Quito }\end{array}$ & Mayor a 4.500 \\
\hline B & $\begin{array}{l}\text { Pontificia Universidad Católica del } \\
\text { Ecuador }\end{array}$ & $\begin{array}{l}\text { Pensión diferenciada, entre } 1900 \text { - } \\
4.000 \text { ( } 30 \text { créditos) }\end{array}$ \\
\hline B & $\begin{array}{l}\text { Universidad Particular } \\
\text { Internacional SEK }\end{array}$ & $3.000-3.600$ \\
\hline C & Universidad de las Américas & $3.000-3.500$ \\
\hline B & Universidad Iberoamericana & $1.600-1800$ \\
\hline C & $\begin{array}{l}\text { Universidad Internacional del } \\
\text { Ecuador }\end{array}$ & $2.500-3.000$ \\
\hline C & $\begin{array}{l}\text { Universidad de Especialidades } \\
\text { Turísticas }\end{array}$ & $1.600-2.000$ \\
\hline
\end{tabular}

Fuente: Sondeo realizado por estudiantes de Ecoturismo. PUCE, 2014.

Elaboración propia 
6.5 Núcleos disciplinares: En las carreras de turismo así como en las de hotelería analizadas, se observa que en el ciclo básico se imparten asignaturas que apoyan los fundamentos tanto del ciclo disciplinar en turismo como del ciclo técnico, la mayoría de las carreras no sobrepasan el $10 \%$ del total de las materias.

Al núcleo disciplinar en turismo le corresponden aquellas materias que interrelacionan al turismo como un fenómeno social y económico, además de asignaturas relacionadas con la hospitalidad del turismo y sus servicios. Todas las carreras analizadas poseen un ciclo disciplinar en turismo que contiene entre el 15 y el $30 \%$ del total de las asignaturas. El núcleo disciplinar técnico es el que contiene entre el 30 y $50 \%$ de las materias de los mesocurrículos analizados. Las carreras de administración y gestión de empresas, tanto turísticas como hoteleras, o combinadas, poseen asignaturas que les permiten a los futuros profesionales administrar un negocio turístico u hotelero; inclusive en aquellas carreras cuya denominación es solo Turismo, el núcleo de materias técnicas es fuerte. Las carreras relacionadas con la Guianza Turística poseen asignaturas que apoyan la interpretación ambiental o cultural, además de la elaboración y evaluación de proyectos turísticos. Otros núcleos disciplinares técnicos, aunque escasos, se enfocan en que los estudiantes puedan realizar planes de negocios y proponer emprendimientos (Tabla No.8).

En las carreras relacionadas con la Administración y/o Gestión Hotelera, el núcleo de asignaturas está orientado a la administración de empresas turísticas y hoteleras; en las carreras relacionadas con la hotelería se reconoce también que existen materias relacionadas con la operación de un hotel para la generación de negocios propios. 
Tabla No.7 Conocimientos, habilidades y actitudes declarados en los perfiles de egreso o profesional de carreras de universidades de las ciudades de Quito, Guayaquil, Cuenca y Riobamba

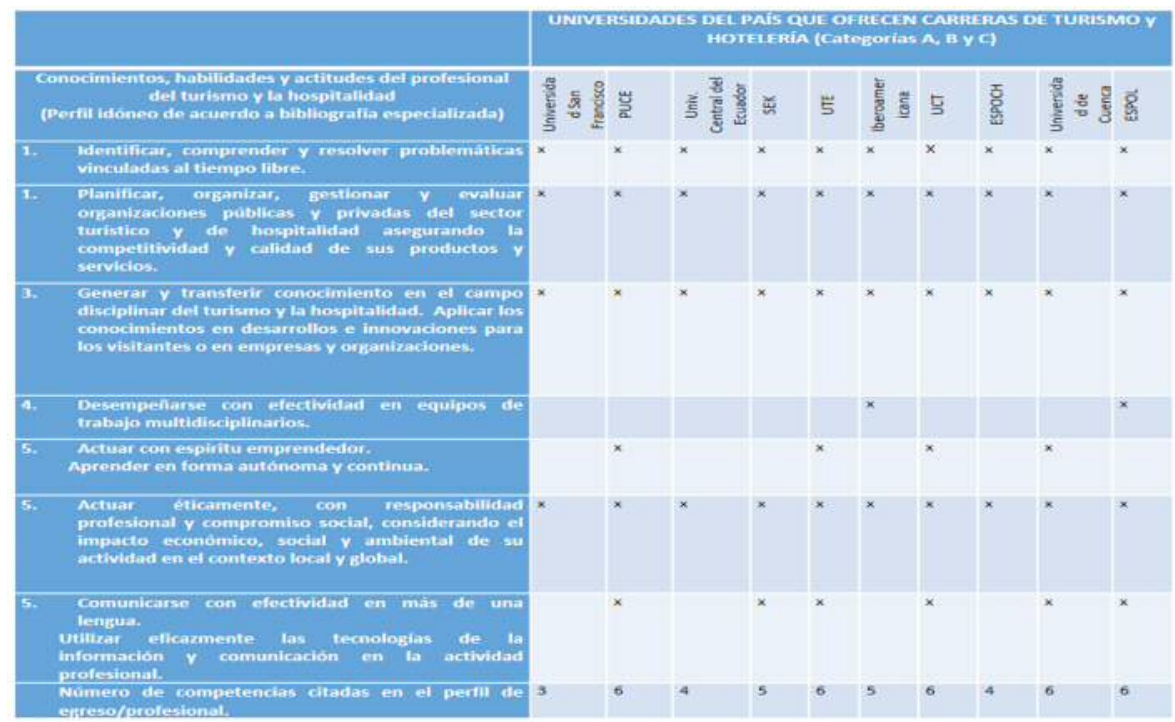

Fuente: Lineamientos de mejora para la formación de recursos humanos en turismo (2014). García Manjón y Pérez López (2008) y Wang, Ayres y Huyton (2009).

Elaboración propia. 
Tabla No. 8 Porcentaje de materias en ciclos disciplinares

\begin{tabular}{|c|c|c|c|c|c|c|}
\hline \multirow{2}{*}{ Universidad Carrera } & \multicolumn{2}{|c|}{ Níxleo dis ciplinar básico } & \multicolumn{2}{|c|}{ Núcleo disciplinar en turismo } & \multicolumn{2}{|c|}{$\begin{array}{l}\text { Núcleo disciplinar técrico en } \\
\text { turismo }\end{array}$} \\
\hline & $\begin{array}{c}10 \% \text { del total } \\
\text { de materias }\end{array}$ & $\begin{array}{l}10 \% \text { del } \\
\text { total de } \\
\text { materias }\end{array}$ & $\begin{array}{c}15-30 \% \text { del total } \\
\text { de } 1 \text { las materias }\end{array}$ & $\begin{array}{l}30-50 \% \text { del total } \\
\text { de las materias }\end{array}$ & $\begin{array}{l}25-30 \% \text { del total } \\
\text { de las materias }\end{array}$ & $\begin{array}{l}30-50 \% \text { del tot } a \\
\text { de } 1 \text { as materias }\end{array}$ \\
\hline \multicolumn{7}{|l|}{$\begin{array}{l}\text { USFQ(Quito y } \\
\text { Guyaquil) }\end{array}$} \\
\hline $\begin{array}{l}\text { Admde Empres as de } \\
\text { Hos pitalid ad }\end{array}$ & $x$ & & $x$ & & & $x$ \\
\hline \multicolumn{7}{|l|}{ PUCE-Quito } \\
\hline Ecoturismo & $x$ & & $x$ & & & $x$ \\
\hline Ges tión Hotelera & $x$ & & $x$ & & & $x$ \\
\hline \multicolumn{7}{|l|}{ Univ: Central } \\
\hline $\begin{array}{l}\text { Turis mo Fis torico } \\
\text { Cultural } \\
\end{array}$ & $x$ & & $\mathbf{x}$ & & & $x$ \\
\hline Turismo ecológico & $x$ & & $x$ & & & $x$ \\
\hline \multicolumn{7}{|l|}{ UCT } \\
\hline $\begin{array}{l}\text { Lic.Guia de Turismo } \\
\text { Nacional }\end{array}$ & $\mathbf{x}$ & & $x$ & & & $x$ \\
\hline $\begin{array}{l}\text { Adm de Empres as } \\
\text { Turis ticas }\end{array}$ & $\mathbf{x}$ & & $x$ & & & $x$ \\
\hline Adm Hotelera & $\mathbf{x}$ & & $x$ & & & $\mathbf{x}$ \\
\hline \multicolumn{7}{|l|}{ SEK } \\
\hline $\begin{array}{l}\text { Adm de Empres as } \\
\text { Turis ticas } \\
\end{array}$ & $\mathbf{x}$ & & $x$ & & & $x$ \\
\hline \multicolumn{7}{|l|}{ UTE } \\
\hline $\begin{array}{l}\text { Adm de empresas } \\
\text { turisticas }\end{array}$ & $\mathbf{x}$ & & $\mathbf{x}$ & & & $\mathbf{x}$ \\
\hline Adm Hotelera & $\mathbf{x}$ & & $x$ & & & $x$ \\
\hline \multicolumn{7}{|l|}{ Iberoamericana } \\
\hline $\begin{array}{l}\text { Admde Empres as } \\
\text { Turis ticas }\end{array}$ & $\mathbf{x}$ & & $x$ & & & $x$ \\
\hline $\begin{array}{l}\text { Adm Empres as } \\
\text { Hoteleras } \\
\end{array}$ & $\mathbf{x}$ & & $x$ & & & $x$ \\
\hline \multicolumn{7}{|l|}{ ESPOCH } \\
\hline Ecoturis mo & & $\mathbf{x}$ & $\mathbf{x}$ & & & $\mathbf{x}$ \\
\hline \multicolumn{7}{|l|}{ UDA } \\
\hline Turis mo & $x$ & & $x$ & & & $x$ \\
\hline \multicolumn{7}{|l|}{ ESPOC } \\
\hline Turis mo & $x$ & & $x$ & & & $x$ \\
\hline
\end{tabular}

Fuente: Páginas web de Universidades que ofertan carreras de turismo y hotelería.

Elaboración propia

Fortalezas observadas: en la ESPOL de Guayaquil se dictan materias en inglés: Ecuadorian Biodiversity, Tour guiding and interpretation, Travel Agencies \& GDS, Tourist product marketing.

En carreras de hotelería llama la atención asignaturas como Auditoria nocturna y Cultura gastronómica. 
La duración de las carreras oscila entre 8, 9 y 10 semestres; existe una relación directa entre la titulación que se otorga con la duración: licenciaturas 8 semestres, ingenierías entre 9 y 10.

6.6 Prácticas pre-profesionales: Se observa como debilidad que 6 de las 10 universidades analizadas, hasta diciembre del 2014, no comunicaron en sus páginas web institucionales las horas de prácticas profesionales que requieren las carreras. En el caso de carreras de turismo: una universidad especifica que los estudiantes requieren realizar 1.500 horas, otra 1.000 horas, dos entre 100-500 y las 6 restantes no especifican.

La información para las carreras de hotelería también es escasa: dos universidades señalan que deben practicar entre 1.000 y 1.500 horas, otras dos entre 100 y 500 y las 6 restantes no especifican.

6.7 Vinculación con la sociedad: Una situación similar ocurre con el número de horas de vinculación con la sociedad: sólo dos universidades informan el número de horas que los estudiantes deben cumplir, tanto para las carreras de turismo como de hotelería: una reporta 50 y otra 100 horas de vinculación con la sociedad.

6.8 Investigación y publicaciones: Tres universidades mencionan, total o parcialmente en la descripción de las carreras, sobre todo de turismo, las investigaciones realizadas y dónde están publicadas. La UCT tiene una revista indexada que se publica semestralmente, el último número fue en octubre del 2013.

\subsection{Postgrados de Turismo en Ecuador}

La Escuela Politécnica del Litoral (2015) ofrece la maestría en Marketing de Destinos y Productos Turísticos. Tiene una duración aproximada de dos años (54.5 créditos). No existe información sobre el precio.

La Universidad de Especialidades Turísticas (2015) oferta la maestría en Gestión del Turismo, con el título profesional de Máster en Gestión del Turismo. Duración 4 semestres. No existe información sobre el precio.

La Universidad Técnica del Norte (2015) brinda una maestría en Eco-Turismo y Áreas Protegidas. No se especifica el título que se obtendrá. La duración es de 4 semestres y un costo de 600 USD por semestre.

La Universidad del Azuay (2015) oferta una maestría en Planificación Turística, el título que se otorga es Magister en Planificación Turística. Dura 2 años y el costo total es de 4.000 USD. 


\subsection{Universidades a nivel internacional reconocidas por sus carreras de turismo}

Universidad de las Islas Baleares (2015): Grado en turismo. El currículo es flexible, presenta un fuerte núcleo disciplinar en turismo, compuesto por asignaturas como: Patrimonio Cultural, Estructura del Mercado del turismo, Recursos Territoriales Turísticos I y II, Análisis cuantitativo de la actividad turística, Economía del Turismo: medición y evaluación del impacto económico del turismo. El núcleo central es la gestión de empresas turísticas. Duración: 4 años. Su fortaleza está en que a partir del 2013 oferta el Grado en Turismo de forma bilingüe: se imparten aproximadamente 10 asignaturas en inglés.

Universidad Rey Juan Carlos (2015): Grado en turismo. Se menciona como fortaleza de esta universidad el que su carrera de turismo posee un cuerpo docente muy cualificado, con docentes de gran experiencia y muy vinculados al sector. Cuenta con una oferta online. Duración 4 años.

Universidad Austral de Chile (2015): Dispone de la Licenciatura en Turismo, en un período de 10 semestres. Los núcleos disciplinares se centran en la administración de empresas turísticas y en la planificación turística a nivel territorial.

Universidad de Sao Paulo (2015): Licenciatura en turismo. En el primer año se destacan asignaturas relacionados con los conocimientos teórico-básicos, se subraya el impacto social, ambiental y económico del turismo. En los siguientes niveles toma importancia la planificación turística asistida por criterios sustentables. Se abordan materias relacionadas con el mercado turístico. Los trabajos realizados por los estudiantes deben proponer soluciones prácticas a los problemas que se presentan en el ámbito del turismo; y para finalizar la carrera tienen que desarrollar un proyecto de investigación sobre aspectos de la problemática turística municipal. Duración 4 años.

Universidad de Ohio (2015): Ofrece grado bachelor en restaurant hotelería y turismo, que es una carrera eminentemente técnica. Los estudiantes son formados para el manejo y la operación de negocios relacionados con el turismo y hotelería.

Universidad Carbondale del Sur de Illinois (2015): Esta universidad acredita un grado de bachelor en Ciencias de la hospitalidad y el turismo. La carrera tiene una perspectiva técnica. El programa posee un núcleo disciplinar fuerte en negocios, y se orienta básicamente a su manejo y operación

Cabe señalar que las universidades estadounidenses ofrecen carreras en Recreación, recreación terapéutica y recreación en ambientes naturales, y que se relacionan con la administración de parques y servicios de recreación e interpretación ambiental. 


\section{Conclusiones}

Como se ha señalado en los resultados de este estudio, en el Ecuador 42 universidades, de 52 vigentes a nivel nacional, ofrecen 117 carreras de turismo y hotelería con una oferta muy heterogénea de titulaciones, sin duda con el objetivo de diferenciarse y captar estudiantes. A pesar de la disimilitud, se observa una clara tendencia hacia la administración y/o gestión de empresas turísticas, turísticas y hoteleras y hoteleras; la oferta de carreras de solo turismo también es considerable. Se otorgan títulos que acogen tanto el grado de licenciatura como el de ingeniería. La heterogeneidad de las carreras y titulaciones puede ser interpretada por la sociedad como una fortaleza, porque existen profesionales especializados en los diferentes ámbitos del turismo, de la hotelería o ambos. Sin embargo, se convierte en una debilidad cuando el profesional no responde de manera solvente a las especificaciones del título profesional otorgado, o está sobrecalificado para los nichos laborales operativos de las empresas turísticas y hoteleras ecuatorianas.

La reestructuración de carreras, de acuerdo con el Reglamento de Régimen Académico (2013) y la oferta de un solo título tanto en turismo como en hotelería (Reglamento de armonización de la nomenclatura de títulos profesionales, 2014), obligan a las universidades a repensar la apertura o no de las carreras en las diferentes provincias: los estudios de oferta y demanda establecerán el porcentaje de egresados y graduados insertos en el ámbito laboral y las necesidades del sector a nivel local. Es necesario recordar que el sector hotelero requiere que las dos terceras partes del talento humano realicen funciones operativas y no gerenciales (SEGIB-OMT, 2009); lo que contradice a la oferta ecuatoriana, ya que la mayoría de carreras tienden a formar profesionales que administren un negocio turístico u hotelero. Es decir, es posible que las universidades formen profesionales para cargos gerenciales, sin conocer si el sector los requiere o no. Así, el resultado es ineficiente y costoso porque profesionales formados para cargos altos están realizando funciones operativas propias de grados tecnológicos, impartidos por institutos u otras instituciones de formación profesional.

La cobertura a nivel nacional tiene sus aristas. Si bien 42 universidades a través de sus matrices y extensiones cubren las 24 provincias del Ecuador; sin embargo Pichincha, Manabí y Guayas concentran el $43 \%$ de la oferta. Esto obedece sin duda a que éstas son las más pobladas del país y por ende tienen una demanda alta, sobre todo Pichincha y Guayas.

La expansión de carreras de turismo y hotelería a nivel nacional se observa como una fortaleza para el sector. Sin embargo, las universidades creadas localmente se encuentran en categoría $\mathrm{C}$ o $\mathrm{D}$, lo que se advierte como una debilidad en lo concerniente a la calidad de la formación profesional. 
En cuanto a la demanda, las universidades con mayor número de estudiantes a nivel nacional son públicas; se debe aclarar, a grosso modo, que son éstas también las que mayor oferta generan. Sin embargo entre la UTE (cofinanciada), la ESPOL (pública) y la UNL (pública) concentran el $22 \%$ de la demanda nacional.

Se puede notar que la UTE, universidad cofinanciada (un porcentaje de su presupuesto es financiado por el Estado y el otro a través del cobro del servicio educativo a los estudiantes), observa un precio que varía entre 1500-2500 USD. Pese a este factor, es la universidad que registra mayor demanda en el país y en Quito; una razón para ello podría ser que es la más antigua en la formación de profesionales en turismo y gastronomía y, gracias a esa experiencia acumulada, es la que mayor demanda reportó entre el 2010 y 2013. Situaciones como esta, hacen ver que la intervención del Estado es necesaria para que el acceso de bachilleres a una educación superior de calidad sea equitativo y justo. La SENESCYT, durante el 2015, informó que existen varios programas que incentivan a jóvenes de escasos recursos y con un buen rendimiento académico (exámenes de ingreso a las universidades) para que accedan a una educación de tercer nivel a través de becas (SENESCYT-ENES, 2015).

Por otro lado, al analizar tanto la oferta como la demanda en Quito, se reconoce que el $74 \%$ de las universidades son privadas, pero el $40 \%$ de éstas se encuentran en categoría $\mathrm{C}$; debilidad que es necesario tenerla en cuenta.

Las universidades de mayor demanda (2010-2013) son: UTE (B), UCE (B), UDLA (C), UFA (A), UCT (C); la PUCE osciló entre el 8-11\% de la demanda total de Quito. Es decir, la categoría de la universidad no ha sido un factor determinante en la demanda: 2 de las 5 universidades mayor solicitadas en Quito se encuentran en la categoría $\mathrm{C}$, hecho que debe ser analizado con más profundidad para evitar conjeturas poco objetivas. Como ya se dijo, uno de los factores determinantes en la elección de la universidad es, sin duda, los precios.

Del análisis cualitativo de los conocimientos, habilidades y actitudes básicas de las carreras de turismo y hotelería, se puede concluir de manera general que las carreras con diferente denominación tienen currículos muy similares, y que carreras con similar denominación pueden ser disímiles en su estructura curricular. Lo que también puede estar influenciado por la duración de las carreras y el título profesional otorgado (Licenciatura o Ingeniería). Se observa como fortaleza que la mayoría de las universidades analizadas mencionan las competencias que, a nivel internacional, se consideran como básicas y transversales para el turismo y los servicios conexos.

De los resultados obtenidos, también se desprende que en el país existe una orientación muy clara a la formación de profesionales para administrar negocios turísticos y 
hoteleros, y guianza en áreas naturales (ecoturismo) o en el ámbito cultural. Los núcleos disciplinares técnicos son perfectamente identificables.

Una debilidad es que en el mesocurrículo no se visualiza el objeto de estudio del turismo, no se distingue una secuencia de asignaturas que expliciten cuáles son los núcleos de aprendizaje y a qué disciplina responden.

Otra debilidad identificada es que cerca del $50 \%$ de las universidades, analizadas en este estudio, no disponen de información, o es parcial, sobre: descripción de programas microcurriculares, precios aproximados, requisitos de graduación como: número de horas de pasantías, número de horas de vinculación con la sociedad, cuerpo docente, campos de especialización, publicaciones, líneas de investigación. Es decir, no facilitan una información relevante para que el potencial estudiante tome decisiones.

De las 42 universidades que ofrecen carreras de turismo y hotelería, 4 ofertan postgrados, y se estima como fortaleza el que éstos estén distribuidos a nivel nacional en cuatro ciudades catalogadas como destinos turísticos del Ecuador: Quito, Guayaquil, Cuenca e Ibarra.

Respecto a la publicación de revistas especializadas indizadas, sólo una universidad a nivel nacional dispone de una revista especializada en turismo con indización; sin embargo, su última publicación es del 2013. Esto viene a ser una falencia y por ende una debilidad de las carreras de turismo a nivel nacional.

En el análisis del currículo de algunas universidades catalogadas de excelencia a nivel internacional, se evidencia de manera general que las IES europeas tienen un currículo orientado al estudio del turismo como fenómeno social, económico, político; lo que se explica por el crecimiento y la importancia que el sector ha tenido en Europa. Sin embargo el núcleo técnico no está descuidado, por lo general tiene una tendencia a la gestión del turismo y servicios conexos. En Sudamérica, resulta interesante la orientación de la Universidad de Sao Paulo, pues mira al turismo como una parte relevante de la economía y de la sociedad de Sao Paulo; los profesionales se forman a través de núcleos problematizadores a los que se intenta dar soluciones viables a través de la investigación. Las carreras que se ofertan en Estados Unidos son esencialmente técnicas, en las que se desarrollan competencias para administrar negocios relacionados con el turismo, hotelería y restauración. 


\section{Recomendaciones}

El turismo para el Ecuador significa un sector que genera divisas (no petroleras) y empleo, entre el 2010 y 2014 se observa un crecimiento económico sostenido. Entonces, la universidad es para el sector turístico un socio estratégico que forma profesionales con capacidades para desarrollarlo en forma cualitativa y cuantitativa.

Para el cambio de la matriz productiva del Ecuador, es imprescindible que existan canales de comunicación abiertos entre el Estado, representado por el gobierno de turno, la academia y el sector productivo-empresarial relacionado con el turismo y los servicios. El Estado ecuatoriano debe apoyar las ideas innovadoras que provengan de la academia, sea pública o privada, y poner a disposición los resultados tanto del sector privado como público. Así mismo, urge la coordinación entre los diferentes organismos del Estado a nivel nacional, provincial y municipal, en procura del manejo eficiente de los recursos económicos y de talento humano.

Los organismos rectores de la educación superior ecuatorianos han dado pasos importantes en lo referente a la normativa para la reformulación de carreras y la unificación de títulos. Sin embargo, no es tarea de las redes de universidades establecer las brechas que existen entre las competencias profesionales específicas que requiere la sociedad, concretamente el sector turístico y hotelero, y las competencias que ofertan las Instituciones de Educación Superior en el Ecuador (IES). Si lo hace el Estado, coadyuvaría a identificar realmente lo que requiere el país. Por ahora, las universidades a través de su cuerpo docente deben reestructurar las ofertas académicas con estudios de pertinencia y de mercado, y otros análisis cuya visión, por ser muy particular y local, no contribuye al desarrollo del sector. De lo contrario, se continuará con la oferta de carreras en turismo con un fuerte componente técnico-administrativo. Se reconoce a nivel mundial que el turismo es una disciplina académica relativamente nueva, y que requiere procesos que contribuyan a visualizar al turismo en un contexto integral y de país. Sólo así las universidades podrán construir, para el ámbito turístico, los núcleos disciplinares que se exigen para entender y dar soluciones creativas que contribuyan al desarrollo humano, ético y sostenible del turismo en el Ecuador.

Por lo expuesto, es recomendable que se reestructure el núcleo disciplinar en turismo en función de los resultados de estudios realizados a nivel latinoamericano, ya que son países con realidades socio-económicas y políticas similares. Este núcleo debe orientarse al análisis del turismo como el conjunto de los diferentes sectores que lo conforman, de los cambios y tendencias del mercado, su relación con los sistemas sociopolíticos que constituyen y regulan la actividad turística, el impacto en las sociedades y en el ambiente, y la planificación de la actividad turística en el territorio (Jafari, 2005). 
El núcleo técnico profesional debe mantenerse en la línea del diseño y evaluación de proyectos turísticos y hoteleros para la propuesta de emprendimientos, sean éstos de interés público o privado, particular o comunitario, con fines sociales o pecuniarios. El desarrollo de competencias, como la innovación, creatividad, aprender en forma autónoma y continua, se hace indispensable.

\section{Referencias Bibliográficas}

Cañibano, C. (Noviembre, 2005). El capital humano: factor de innovación, competitividad y crecimiento. En Francisco. Iribarren (Vicepresidente y Consejero de Hacienda) Sexto Congreso de Economía de Navarra. Congreso llevado a cabo en la Universidad de Navarra, Pamplona España.

Consejo de Educación Superior (CES). (2013). Reglamento de Régimen Académico. CES. Recuperado de: http://www.ces.gob.ec/2012-10-29-08-51-25/2012-10-29-08-5154

Consejo de Educación Superior (CES). (2013). Resolución No.001-073CEAACES-2013. Categorización de Universidades y Escuelas Politécnicas. Recuperado de: http://www.ces.gob.ec/ies/universidades-y-escuelas-politecnicas/por-categoria/ pregrado/categoria-a/169-universidad-de-las-fuerzas-armadas-espeO

Consejo de Educación Superior (CES). (2014). Reglamento de armonización de la nomenclatura de títulos profesionales y grados académicos que confieren las Instituciones de Educación Superior del Ecuador. CES. Recuperado de: http://www.ces.gob.ec/201210-29-08-51-25/2012-10-29-08-51-54.

Consejo de Evaluación, Acreditación y Aseguramiento de la Calidad de la Educación Superior, (CEAACES) (2013). Suspendida por falta de calidad. El cierre de catorce universidades en Ecuador. CEAACES. Quito, Ecuador:

Escuela Politécnica del Litoral (2015). Maestría en Marketing de Destinos y Productos Turísticos. ESPOL. Recuperado de: http://www.fimcbor.espol.edu.ec/maestria_mdpt

García Manjón, J.V., Pérez López. MC. (2008). El grado de turismo: un análisis de las competencias profesionales. Cuadernos de Turismo. 21, pp. 67-83. Universidad de Murcia España. Recuperado de: http://www.redalyc.org/articulo.oa?id=39802103.

Instituto Superior de Shanghái Jiao Tong (2015). World University Rankings. Universidad de China. Recuperado de: (2015)http://www.timeshighereducation.co.uk/ world-university-rankings/2014-15/world-ranking/region/south-america.

Jafari, J. (2005). El turismo como disciplina científica. Política y Sociedad, 42 (1), pp 3956. Recuperado de https://docs.google.com/document/d/13BLXe6PYap7w7m7Jiznb8qKzx7x7sB31VAqEueg4C4/edit

Larrea de Granados, E. (2014). El Currículo de la Educación Superior desde la Complejidad Sistémica. CES. Recuperado de: http://www.google.com.ec/url?sa=t $\& r c t=j \& q=\&$ esrc $=$ s\&source $=$ web $\& \mathrm{~cd}=2 \& v e d=0 \mathrm{CCIQFjAB} \& u r l=h t t p \% 3 \mathrm{~A} \% 2 \mathrm{~F} \% 2$ Fwww.ces.gob.ec\%2Fregimen-academico\%2Fplan-de-acompanamiento\%2Ftaller- 
dia-01\%3Fdownload\%3D609\%3 Ael-curriculo-de-la-educacion-superior-desde-lacomplejidad-sistemica\&ei=dG8LVe_LHYqyggT6vICADA\&usg=AFQjCNEPx5_uXhd VLiy5kEYVKDBNgLmXbw\&sig2=G45VrhgjiqWqfejFziCNfA

Ministerio de Turismo del Ecuador (MINTUR). (2015). Anuario de Estadísticas Turísticas. Boletín de Estadísticas Turísticas 2009-2013. Recuperado de: http://servicios. turismo.gob.ec/index.php/portfolio/turismo-cifras/175-anuario-de-estadisticas-turisticas Ministerio de Educación de Argentina, Ministerio de Turismo de Argentina. (2014). Lineamientos de Mejora para la Formación de Recursos Humanos en Turismo. Recuperado de: http://www.uipt.net/upload/Documento\%20SPUTurismo\%20v2.0.pdf

Muñoz de Escalona, F. (2010). Epistemología del turismo. Un Estudio Múltiple. Revista de Investigación en Turismo y Desarrollo Local. 3 (7). Recuperado de http://www.eumed. net/rev/turydes/07/index.htm

Niding, M. Andueza, J., Farías, D., Alonso, MA. \& Zamudio, A. (2011). Los obstáculos Epistemológicos del Turismo como dominio del saber. Tiempo Libre Turismo y Recreación. 1. Universidad Nacional de Mar del Plata. Recuperado de http://nulan.mdp. edu.ar/1757/1/Apo2011a15v1pp13-38.pdf

Organización Mundial del Turismo (OMT). (2014). Panorama OMT del Turismo Internacional. OM. Edición 2014. Recuperado de: http//www.unwto.org/pub

Osorio, F., Maldonado, D., Rodriguez, C. (2012). Calidad de la educación básica y media en Colombia: diagnóstico y propuestas. Colombia. Universidad del Rosario. Serie Documentos de Trabajo 126.

Rama, Claudio (2006). "Introducción". Informe sobre la educación Superior en América Latina y el Caribe 200-2005. Caracas: IESALC-UNESCO, p.1-18.

Ramírez Gallegos René (2013). Tercera ola de transformación de la Educación Superior en Ecuador. Quito: Secretaría Nacional de Educación Superior, Ciencia, Tecnología e Innovación.

Ring, A., Dickinger, A. \& Wöber, K. (2009). Designing the Ideal Undergraduate Program in Tourism. Journal of Travel Research, 48 (1), 106-121.

Secretaría Nacional de Educación Superior Ciencia Tecnología e Innovación (SENESCYT) (2015). Número de estudiantes matriculados durante los años 2010-2013 por universidades a nivel Nacional. SNIESE. SENESCYT. Quito.

Secretaría Nacional de Planificación y Desarrollo. (SENPLADES) (2012). Transformación de la Matriz Productiva. Recuperado de http://www.planificacion.gob. ec/wp-content/uploads/downloads/2013/01/matriz_productiva_WEBtodo.pdf.

Tribe, J. (2005). Tourism, Knowledge and the Curriculum. An Handbook of Tourism Education. Airey, D., Tribe, J. Ed. An Handbook of Tourism Education. (pp. 47-60). Elsevier.

Universidad Austral de Chile (UACH). (2015). Administración Empresas de Turismo. Recuperado de: https://www.uach.cl/admision/principal/valdivia/administracionempresas-de-turismo.

Universidad del Azuay (UDA). (2015). Maestría en Planificación Turística. UDA. 
Recuperado de: http://www.uazuay.edu.ec/maestrias/turismo.pdf

Universidad de Baleares (2015). Grado de Turismo. Recuperado de: http://estudis.uib. es/es/grau/turisme/GTUR-P/

Universidad Carbondale del Sur de Illinois (2015). Bachelor en Hospitality and Tourism. Recuperado de: http://hospitality-1st.com/PressNews/SIU-HTM-IL.html Universidad de Especialidades Turísticas (UCT). (2015) Maestría en Gestión del Turismo. UCT. Recuperado de: http://www.uct.edu.ec/index.php/unidades-academicas/ escuela-de-posgrados

Universidad de Ohio (2015). Carrera de Restauración, Hotelería y Turismo. Recuperado de: http://www.ohio.edu/majors/undergrad/cehs/hcs/rht.cfm

Universidad Rey Juan Carlos. (2015). Grado de Turismo. Recuperado de: http://www. urjc.es/estudios/grado/turismo/turismo.html\#

Universidad de Sao Paulo (USP). (2015). Grado de Turismo. Recuperado de: https:// uspdigital.usp.br/jupiterweb/listarGradeCurricular? codcg $=27 \&$ codcur $=27531 \& \operatorname{codhab}=$ $4 \&$ tipo $=\mathrm{N}$

Universidad Técnica del Norte (UTN). (2015). Maestría en Eco-Turismo y Áreas Protegidas. UTN. Recuperado de: http://www.utn.edu.ec/postgrado/

Vandam, M., P. (2013). Análisis de los planes de estudio de carreras de grado en turismo y hotelería en el ámbito universitario en América. Universidad de Kennedy. Repositorio digital del Ministerio de Turismo Argentino.

Wang, J., Ayres, H., Huyton, J. (2009). Job ready graduates: a tourism industry perspective. Journal of Hospitality and Tourism Management, 16 (01) pp 62-72.

World Economic Forum (2014). Travel and Tourism Competitiveness. Recuperado de: http://www.weforum.org/issues/travel-and-tourism-competitiveness 